\title{
CRecimiento eCONÓMico y Distribución DEL INGReso EN PERÚ
}

\section{Luis Varona-Castillo y Jorge Ricardo Gonzales-Castillo ${ }^{\mathrm{a}}$}

Fecha de recepción: 30 de abril de 2020. Fecha de aceptación: 17 de noviembre de 2020.

$$
\text { https://doi.org/10.22201/iiec.20078951e.2021.205.69636 }
$$

\begin{abstract}
Resumen. En el presente artículo se analizan los determinantes causales de la distribución del ingreso en Perú (DIP) durante el periodo 1985-2017. Se cuenta con evidencia empírica que apoya la hipótesis sobre que el nivel del PIB per cápita y el crecimiento económico ralentizado y no sostenido de la economía ha empeorado la DIP, reflejada en una relación de causalidad en forma de W, no cumpliéndose la hipótesis de U-invertida de Kuznets, que refleja una economía dual con diferencias de productividad, bajos niveles de capital humano y debilidad institucional. Se recomienda equilibrar la relación mercado-Estado-sociedad con políticas de economía de libre mercado y no rentistas, políticas de inversión en capital humano, innovación y tecnología, fortalecimiento del capital social -en la dirección del crecimiento económico endógeno- del desarrollo humano inclusivo y sostenible.
\end{abstract}

Palabras clave: Perú; crecimiento económico; distribución del ingreso; coeficiente Gini; capital humano 1985-2017.

Clasificación JEL: C51; O11; O15; O41; O47.

\section{Economic Growth ANd Income distribution In Peru}

\begin{abstract}
This article analyzes the causal determinants of income distribution in Peru (IDP) during the period 1985-2017. There is empirical evidence suggesting that the GDP per capita and the slowed and unsustained economic growth of the economy have negatively impacted IDP. This can be conceptualized as a W-shaped causal relationship, in contrast to Kuznets' inverted-U hypothesis, thus reflecting a dual economy with productivity differences, low levels of human capital, and institutional weakness. This article contributes to the debate by making several recommendations, namely: balancing the market-state-society relationship with free-market and non-rentier economic policies, human capital investment policies, innovation and technology, and strengthening the social capital (geared towards endogenous economic growth) of inclusive and sustainable human development.
\end{abstract}

Key Words: Peru; economic growth; income distribution; Gini coefficient; human capital 1985-2017.

a Universidad Nacional de Piura, Perú. Correos electrónicos: lvaronac@unp.edu.pe y jgonzalesc@unp.edu.pe, respectivamente. 


\section{INTRODUCCIÓN}

Los hechos estilizados muestran la reducción de la pobreza en Perú cercana al 34\% respecto al nivel de 1985 . No obstante, la distribución del ingreso en Perú (DIP) ha empeorado al aumentar en promedio el coeficiente Gini (Gini) en $0.11 \%$. Este escenario se presenta en el periodo 1985-2018, en el que la tasa de crecimiento económico promedio anual es lento (1.98\%).

Según Kuznets, la distribución del ingreso (DI) debería empeorar al inicio del desarrollo económico y una vez empleada la fuerza de trabajo en el sector industrial, con elevados salarios, disminuir la desigualdad como un proceso natural (Kuznets, 1955 y 1963). Sin embargo, Piketty (2014) considera necesaria la intervención del Estado para llegar a la equidad en la DI.

Para Perú, desde el trabajo seminal de Figueroa y Webb (1975) y, posteriormente, Figueroa (1973, 1993, 2003 y 2010), Mendoza et al. (2011), Tello (2011), Yamada et al. (2016), Seminario y Zegarra (2016), Cruz-Saco et al. (2018) y, recientemente Alarco et al. (2019), coinciden en presentar evidencia histórica sobre la desigualdad en la DIP.

Las políticas económicas implementadas en el periodo 1985-1990 y 19912017, no solucionaron la DIP. La Estrategia de Desarrollo de Industrialización por Sustitución de Importaciones (EDISI) - planificada por el Estado- dio paso a la Estrategia de Desarrollo Neoliberal (EDNL), donde prima el libre mercado. En ambos modelos no se concreta una reversión sostenida de la DIP. Se plantean entonces las siguientes preguntas: ¿qué causas explican la DIP? ¿Cuál es el impacto del crecimiento económico y el nivel capital humano en la DIP? ¿El crecimiento económico reduce la DIP o hay crecimiento sin equidad?

El objetivo de la presente investigación es analizar los determinantes causales de la Dip, en el periodo 1985-2017. El artículo se estructura en siete secciones. La segunda sección corresponde a la revisión de la literatura; mientras que la tercera muestra los hechos estilizados. La cuarta sección presenta la metodología empleada. La quinta muestra los principales resultados y la sexta la discusión, para finalmente llegar a la séptima sección de las conclusiones.

\section{REVISIÓN DE LA LITERATURA}

La relación causal entre crecimiento económico y Di sigue siendo debatida teórica y empíricamente. Para los clásicos, como Smith (1776), es la especialización por la división del trabajo la que genera el incremento de la producción y de la productividad que, por un lado, genera mayores salarios y mayor 
consumo para los trabajadores e ingresos para la sociedad y que, por otro lado, genera mayores beneficios para los capitalistas, permitiendo ahorrar, invertir y acumular capital, obteniendo así mayor ingreso, riqueza y crecimiento económico para una nación. Este proceso de generación de salarios y beneficios se reproduce en los mercados, que guiados como por una "mano invisible" lleva a los individuos, que busca sus propios intereses, a obtener beneficios para un país, lo que implica que el mercado endogenice la DI en salarios y beneficios entre trabajadores y capitalistas.

Para Ricardo (1817), la Di se caracteriza por mayores rentas por alquiler de la oferta fija de tierras en detrimento de salarios de trabajadores y de los beneficios de capitalistas. Marx (1867) considera un salario de subsistencia para los trabajadores dado el "ejercicio industrial de reserva" o desempleo, que no está dado por la productividad marginal de la mano de obra, sino que puede ser político e institucional. La apropiación de la plusvalía no reflejada en el salario es considerada como beneficio que se destina a la inversión y acumulación de capital, afectando el crecimiento económico y que empeoran la DI.

Marshall (1890) considera que la oferta y la demanda en los mercados determinan los precios de bienes, servicios y factores productivos, por ende, el salario y la tasa de interés lo hacen en los mercados de trabajo y de capital, basado en su productividad marginal, afectando endógenamente a la asignación de factores productivos y DI. Sostiene que la intervención del Estado con salarios mínimos en mercados de trabajo distorsiona la asignación de recursos en la economía y genera desempleo, afectando negativamente el crecimiento económico y la DI.

Poskeynesianos como Kaldor (1956), Kalecki (1954) y Pasinetti (1962) consideran la DI como función de la demanda agregada. Sostienen que los asalariados, dada su alta propensión al consumo, gastan una gran proporción del ingreso en consumo, y los capitalistas, dada su alta propensión al ahorro, lo canalizan a la inversión y acumulación de capital, en suma se expande por ambos lados la demanda agregada. Así, la desigual Di entre capitalistas y asalariados determina el nivel y la composición de la demanda agregada. Por ende, la DI aparece endógenamente resultante de la demanda agregada y del crecimiento económico.

Para Lewis (1954) existen salarios de subsistencia dada la sobrepoblación y, por lo tanto, la desigual Di es endógena y se explica por los crecientes beneficios de los capitalistas, que son invertidos y acumulados, y considerados como el motor del crecimiento económico; así como por los estancados salarios de subsistencia. En el largo plazo, establece que los salarios se incrementarán y pagarán de acuerdo con su productividad marginal, mejorando la desigual DI, 
cuando se elimine la sobrepoblación o desempleo y, por ende, se elimine la dualidad de la economía por el crecimiento económico.

Un trabajo pionero que presenta evidencia empírica y contrasta la relación entre el crecimiento económico y la DI es el de Kuznets (1955), que encuentra para Estados Unidos, Inglaterra y Alemania un empeoramiento de la DI en las primeras etapas de desarrollo para luego mejorar a partir de un punto de inflexión. Señala la existencia de una relación de Hipótesis de U-invertida de Kuznets (HUK) entre ambas variables, que implica que existe un empeoramiento de la DI en las primeras etapas del desarrollo en una economía agrícola, que luego se revierte mejorando la DI con el crecimiento económico, tendiendo a la equidad en DI automáticamente con el mercado. Según el autor, la Di obedece a imperfecciones de mercados de trabajo, así como al cambio de estructura productiva y diferencias tecnológicas y de productividad.

Estudios posteriores encuentran evidencia empírica que apoya o no una relación entre la DI y el ingreso per cápita o crecimiento económico (Ahluwalia, 1976; De Gregorio y Lee, 2002; Deininger y Squire, 1998; Forbes, 2000; Gonzales-Castillo, 2009; Guiga y Rejeb, 2012; Heshmati, 2004; Knowles, 2005; Kuznets, 1963; Panizza, 2002; Papanek y Kyn, 1986; Ravallion, 2001; White y Anderson, 2001; Zanzzi et al., 2018).

Dichos estudios consideran, además del crecimiento económico, otros determinantes causales de la DI. Ahluwalia (1976) explica la relación entre capital humano (educación) y su rol como mecanismo igualador de ingresos. Sostiene que la inversión en capital humano expande capacidades, mejora destrezas y aptitudes que incrementan su productividad e ingresos personales, por lo que, promueve la igualdad de ingresos en el largo plazo. Asimismo, De Gregorio y Lee (2002) encuentran evidencia empírica de una relación negativa y estadísticamente significativa entre logro educativo y la DI para países de diversas regiones del mundo.

Asimismo, hay autores que consideran relevante la tecnología, como Foellmi y Zweimüller (2017), que sostienen que los círculos viciosos vía desigualdad de innovaciones a través de un efecto de precio y un efecto de tamaño de mercado generan desigualdad en la Di. Perera-Tallo (2017) confirma que la desigualdad está en aumento en muchos países, asociada al cambio tecnológico. Señala que el cambio tecnológico incrementa la participación del factor menos distribuido equitativamente, agudizando la desigualdad a lo largo de la ruta de crecimiento.

Para Bourguignon y Morrisson (1990) son las dotaciones en los recursos minerales y educación secundaria los determinantes en la DI. Mientras que para Forbes (2000) es la educación femenina y masculina y el gasto público 
en educación. De Gregorio y Lee (2002) consideran que la educación convierte el crecimiento económico en más igualitario. Por su parte, Contreras y Ffrench-Davis (2012) recomiendan profundas reformas microeconómicas, que implica el progreso en la calidad de la educación y apoyo público a la innovación para mejorar la DI. Cheng y Wu (2016) investigan la DI en China y encuentran que esta relación fue impulsada, por baja productividad, en la agricultura en relación con la economía, la educación secundaria y superior. Para Chong y Gradstein (2017), la Di es el efecto conjunto de las desigualdades políticas y económicas sobre los impuestos redistributivos y la calidad institucional.

Otros autores cuestionan la estructura del modelo capitalista por los resultados perversos en DI (Alvaredo et al., 2013; Piketty, 1995, 2014 y 2015; Piketty y Saez, 2013). Piketty (2014) concluye que existe robusta evidencia en el capitalismo mundial de una tendencia a mayor concentración de riqueza y altos niveles de desigualdad en la DI, los cuales revelan la única ley que rige el capitalismo: la tasa de retorno del capital " $r$ " es mayor que la tasa de crecimiento económico " $g$ ".

Existe una gran diversidad de estudios de corte trasversal y de panel data. Sin embargo, son escasos los estudios de series de tiempo sobre los determinantes de DI y su relación con el crecimiento económico, sobre todo, en economías en desarrollo o países de América Latina (AL). Uno de los trabajos que encuentra evidencia empírica que prueba la existencia de desigualdad a través de la HuK en Brasil es el de Keller (2012), ya que muestra que el capital humano explica negativamente la DI.

Ranis (1980), para Colombia, entiende que la DI es una medida del éxito o fracaso de una sociedad. Para ello se plantea que antes del resolver el crecimiento económico éste debería ser equitativo. Afirma que el crecimiento y la DI están relacionados y deben ser explicados de manera simultánea en cualquier contexto teórico. En otras palabras, la medida de DI no debe verse expost de la equidad con que el producto se generó y distribuyó, sino como una parte integral de cualquier teoría positiva del desarrollo.

Ranis et al. (2000) encuentran conexiones entre crecimiento económico y desarrollo humano para una muestra más global de países. Las regresiones entre países evidencian una relación significativa en ambas direcciones, con el gasto público en salud y educación; y la tasa de inversión y la distribución de ingresos significativos. Lo anterior da lugar a círculos virtuosos o viciosos, donde el buen o mal desempeño en desarrollo humano y crecimiento económico se refuerzan mutuamente. Ranis y Stewart (2002) replican la metodología anterior a un grupo de países de AL y los resultados refuerzan las 
trayectorias de crecimiento económico y de DI. De acuerdo con las cuatro categorías de clasificación que sugiere, Perú sería un país que pasó de un ciclo vicioso de crecimiento hacia desarrollo humano en el periodo de 1960-1990, por uno más reciente de 1990-2000, de ciclo virtuoso de desarrollo humano hacia crecimiento económico.

Wahiba y El Weriemmi (2014) presentan evidencia empírica indicando que el crecimiento económico agrava la desigual DI en Túnez y que los efectos son acelerados por el proceso de liberalización del comercio. Encuentran que el capital humano y el desarrollo financiero tienden a contrarrestar los efectos perversos del crecimiento económico al mejorar la DI.

Nwosa (2019) encuentra evidencia empírica de un impacto positivo del crecimiento económico sobre la desigualdad en la DI en Nigeria. Señala que el nivel de PBI per cápita afecta positivamente a la DI, pero no es estadísticamente significativo. Asimismo, encuentra que el gasto público y la población tienen impactos directos sobre la DI, mientras que la apertura externa tiene un impacto negativo sobre la DI.

En un estudio para Chile, Zanzzi et al. (2018) encuentran evidencia de HUK, tanto en niveles como en tasas de crecimiento. Asimismo, se muestra una relación negativa entre pobreza y la DI, mientras que el capital humano presenta una relación positiva, empeora la DI, aunque es estadísticamente no significativa.

En Perú, Figueroa y Webb (1975) analizan el impacto de las políticas y reformas estructurales efectuadas por el gobierno del general Juan Velasco (1968-1975). Sostienen que no se evidencia mejora significativa para los muy pobres, tanto por el crecimiento del ingreso nacional como por los beneficios de las medidas redistributivas del gobierno. Para Figueroa (1993 y 2003), la economía peruana está en desarrollo y los modelos económicos clásico, neoclásico y keynesiano no la explican. Afirma que existe una economía capitalista y una economía campesina. Plantea que la economía peruana es un sistema estable, pero incapaz de eliminar el dualismo y la DIP. Figueroa (2010) sostiene que la educación no es un sistema nivelador de los ingresos y se explica por dos factores: la desigualdad en la dotación inicial de la distribución de activos económicos y políticos entre grupos sociales y la dotación de factores de la sociedad. Así, estos factores exógenos hacen que el sistema educativo siga siendo no nivelador y genere desigual DIP.

Tello (2010 y 2011) enfoca el tema de DIP desde la preocupación por el sector informal o economía dual, en el cual se genera gran parte de los ingresos de la población. Sostiene que las mejoras del sector informal podrían generar 
una menor desigualdad de DIP. Y que el crecimiento económico no genera un decrecimiento del tamańo del sector informal.

Para Alarco et al. (2019), la desigual DIP se explica por la relación causal positiva de la remuneración mínima vital e inflación; mientras que los términos de intercambio y precio del petróleo Brent tienen un impacto negativo.

Finalmente, con base en la teoría económica y evidencia empírica, se puede concluir y seleccionar como variables causales sobre la desigual DI a: nivel de PBI per cápita, crecimiento económico, capital humano (educación), dotación de recursos naturales (RR.NN.), estructura productiva, mecanismos de transferencias y políticas tributarias, desarrollo financiero, demanda agregada (gasto público, ahorro e inversión, exportaciones) e institucionalidad y diferenciales de productividad en el que la tecnología juega un papel importante. La evidencia empírica sobre las relaciones de causalidad de los determinantes de la DI no es concluyente ni robusta en economías en desarrollo.

\section{HECHOS ESTILIZADOS}

Existe un persistente comportamiento cíclico de la desigual DIP. La figura 1 muestra la evolución del coeficiente Gini en el periodo 1985-2017, cuyo valor oscila entre 0.59 y 0.67 , con periodos de aumento y otros de reducción y con un promedio anual de 0.63 , lo que implica que Perú presenta una desigual DI muy superior a países de dinámico crecimiento como son Corea del Sur y Taiwán (0.28 y 0.38, respectivamente, según Korzeniewicz y Moran, 2005).

Se observa que el coeficiente de Gini oscila alrededor de su media de 0.63 , con una tendencia decreciente y es integrada de orden cero, I(0). Asimismo, el nivel de PBI per cápita oscila alrededor de su media de US\$3 979, y es integrada de orden cero $\mathrm{I}(0)$, y presenta una tendencia decreciente en el periodo 1987-1992 y una tendencia creciente en el periodo1993-2017.

En la figura $2 \mathrm{~A}$ parecería que ambas variables van juntas y manifiestan una relación de largo plazo. Además, en la figura 2B la evolución volátil de las tasas de crecimiento de ambas variables permite inferir que ni el crecimiento económico ni la tasa de crecimiento del Gini son sostenibles. En el lento crecimiento económico promedio anual de $1.98 \%$ y tendencia a la desigualdad con un crecimiento promedio anual de Gini $0.11 \%$, juegan un rol causal las políticas fiscales y monetarias expansivas de demanda que generaron hiperinflación y recesión; caída de precios internacionales de minerales; crisis financiera (2008), y políticas públicas que no mitigan la incidencia de los grupos de poder oligopólicos y debilidad institucional. 
Figura 1. Evolución, tendencia y ciclo del coeficiente Gini, periodo 1985-2017. Año base $2010=100$

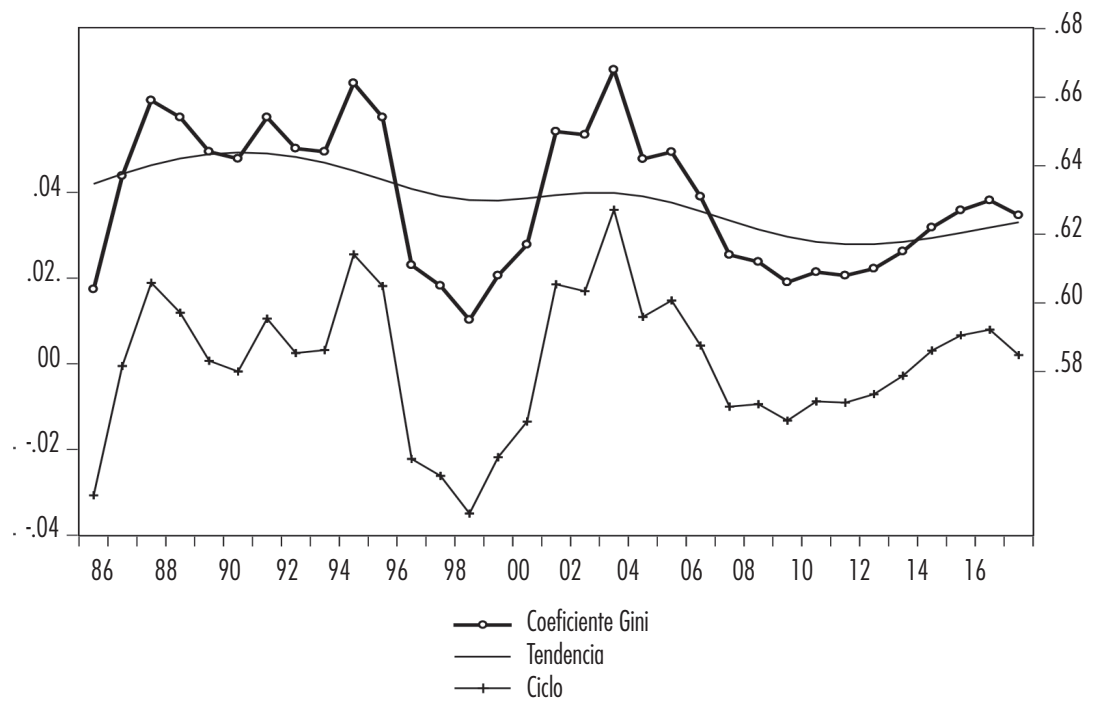

Fuente: elaboración propia. Se aplicó filtro Hodrick-Prescott-FHP.

La tabla 1 muestra estadísticas descriptivas de las dos variables relevantes para cuatro periodos de análisis. Se identifica -como hechos estilizados- que la economía peruana, en el periodo 1985-2017, presenta un relativo bajo ingreso per cápita promedio anual de US\$3 979, mientras que el coeficiente de Gini fue 0.63, mostrando que existe una desigual DI, y observando además que el ingreso per cápita por su coeficiente de variación (28.6) fue mucho más volátil que Gini (3.3). Asimismo, las volatilidades se reducen paulatinamente para ambas variables tendiendo a mejorar la DIP, excepto el periodo de bonanza por exportación de RR.NN. (2002-2009), en el que paradójicamente se incrementó el nivel PBI per cápita y empeoró la DIP, contrario a lo observado en la mayoría de los países de AL en dicha década.

Perú, en el periodo 1985-2017, se identifica como hecho estilizado un comportamiento volátil o no sostenido, tanto para el crecimiento económico como para la DIP, siendo mayor la volatilidad del crecimiento económico que del Gini en casi todos los periodos. 

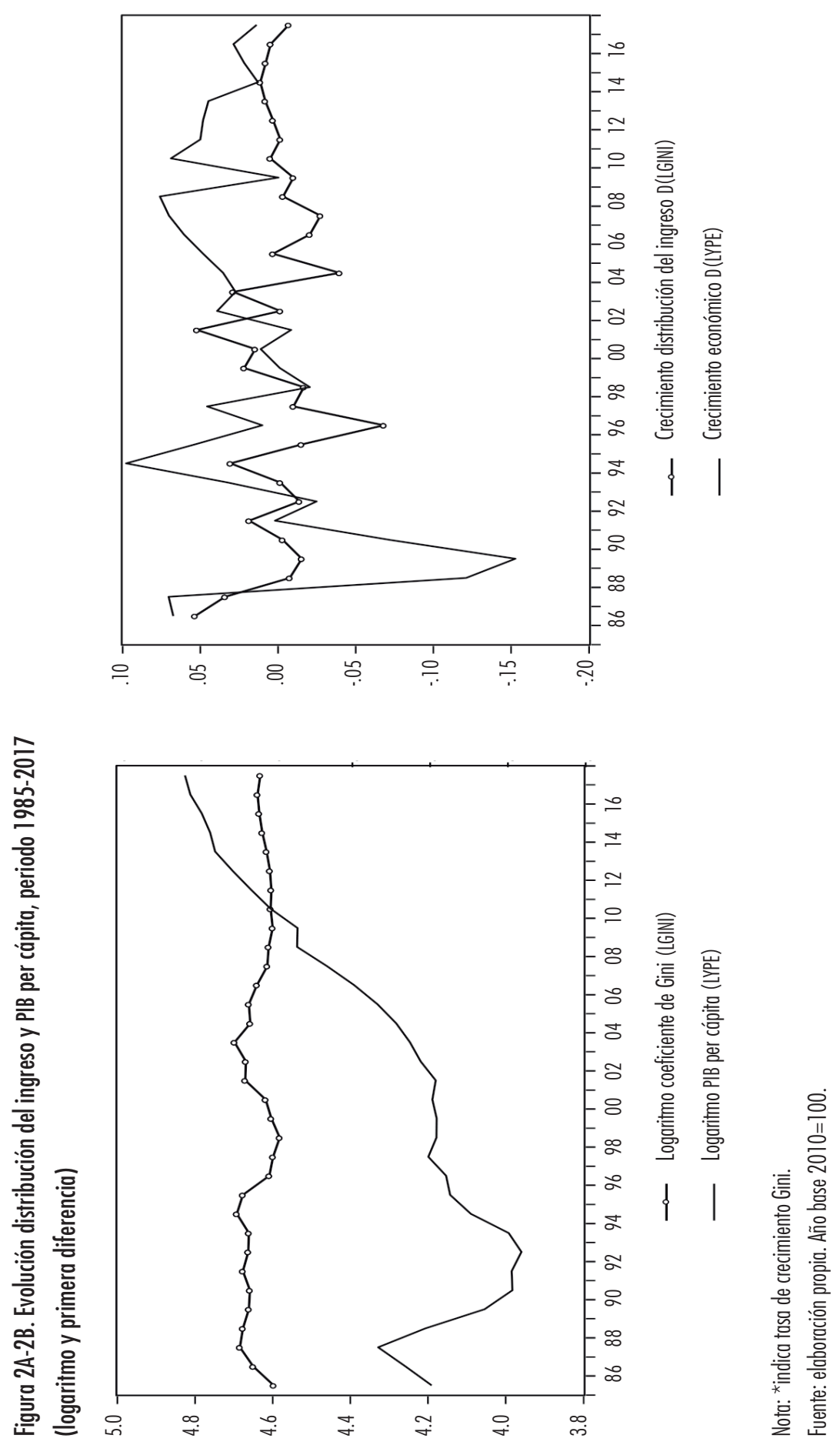
Luis Varona-Castillo y Jorge Ricardo Gonzales-Castillo

Tabla 1. Estadísticas descriptivas del PIB per cápita y coeficiente Gini (nivel y primera diferencia) 1985-2017

\begin{tabular}{lcccccccccc}
\hline NIVEL & YPCS & CG & YPCS & CG & YPCS & CG & YPCS & CG & YPCS & CG \\
\hline Media & 3979.0 & 0.6 & 3112.8 & 0.6 & 3165.9 & 0.6 & 4007.1 & 0.6 & 5731.8 & 0.6 \\
$\begin{array}{l}\text { Desviación } \\
\text { estándar }\end{array}$ & 1136.9 & 0.02 & 446.4 & 0.02 & 198.9 & 0.02 & 508.5 & 0.02 & 430.9 & 0.01 \\
Coef. variación & 28.6 & 3.3 & 14.3 & 2.8 & 6.3 & 3.5 & 12.7 & 3.5 & 7.5 & 1.5 \\
Observaciones & 33 & 33 & 8 & 8 & 9 & 9 & 8 & 8 & 8 & 8 \\
\hline TCE & DLYPCS & DLCG & DLPCS & DLCG & DLYPCS & DLCG & DLYPCS & DLCG & DLYPCS & DLCG \\
\hline Media & 1.98 & 0.11 & -3.29 & 0.94 & 2.45 & 0.08 & 4.45 & -0.88 & 3.62 & 0.40 \\
Desviación & 5.40 & 2.44 & 8.72 & 2.65 & 3.68 & 3.46 & 2.49 & 2.10 & 2.02 & 0.58 \\
estándar & & & & & & & & & & \\
Coef. variación & 272.0 & 2218.1 & -265.0 & 281.0 & 150.5 & 4325.0 & 55.8 & -238.6 & 55.8 & 145.0 \\
Observaciones & 32 & 32 & 7 & 7 & 9 & 9 & 8 & 8 & 8 & 8 \\
\hline Periodo & $1985-2017$ & $1985-1992$ & $1993-2001$ & $2002-2009$ & $2010-2017$ \\
\hline
\end{tabular}

Nota: indica coeficiente de Gini.

Fuente: elaboración propia.

Se observa también que en el periodo 1985-1992, la tasa de crecimiento económico promedio anual fue $-3.29 \%$, asociada a un empeoramiento de la DIP con una tasa de crecimiento del Gini de 0.94 , siendo más volátil el crecimiento económico. En el periodo 2002-2009 se observa lo contrario con una tasa de crecimiento económico promedio anual de $4.45 \%$, asociada a un mejoramiento de la DIP con una tasa de crecimiento de Gini de $-0.88 \%$, siendo más volátil la DIP, en un entorno económico del boom de exportación de RR.NN.

La figura 3A muestra la correlación negativa y estadísticamente significativa en niveles entre LGINI y LYPE $(r=-42 \%)$. La figura $3 \mathrm{~B}$ en tasas de crecimiento presenta una asociación positiva y estadísticamente no significativa entre DLGINI y DLYPE $(r=18 \%)$.

En la figura $4 \mathrm{~A}$ y $4 \mathrm{~B}$ se observa la relación dinámica entre el РBI per cápita y Gini en niveles y tasas de crecimiento, indicando que más que HUK, parecería habría una curva W, de la desigual DIP (HCW) asociada a los ciclos de crecimiento económico no sostenido y desigual DIP. 


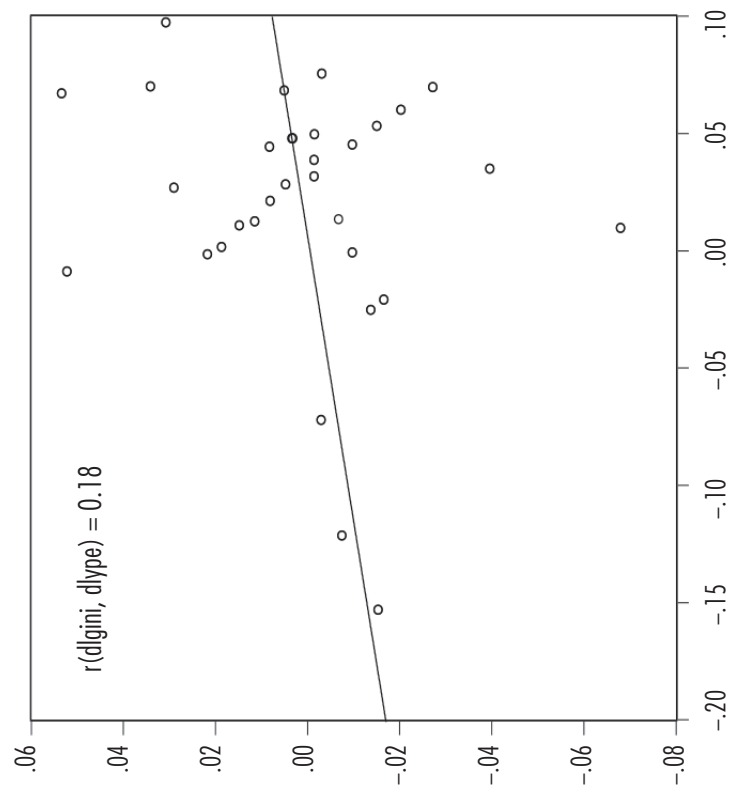

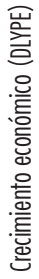

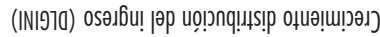

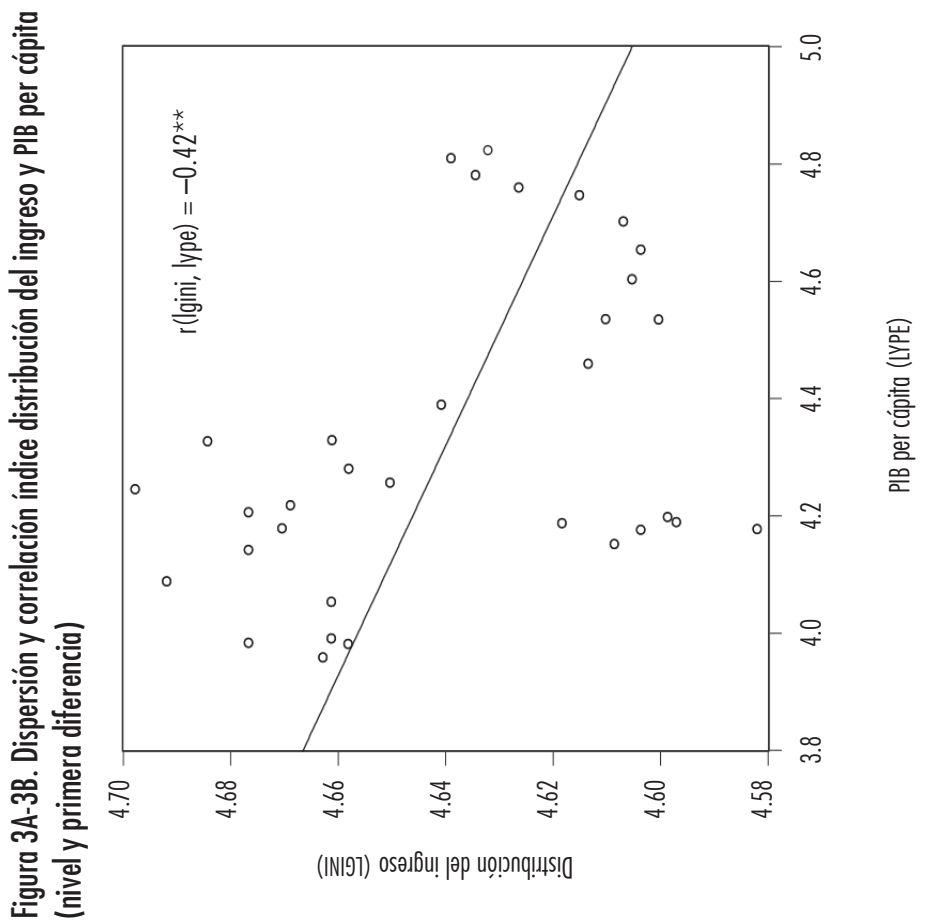

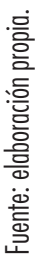




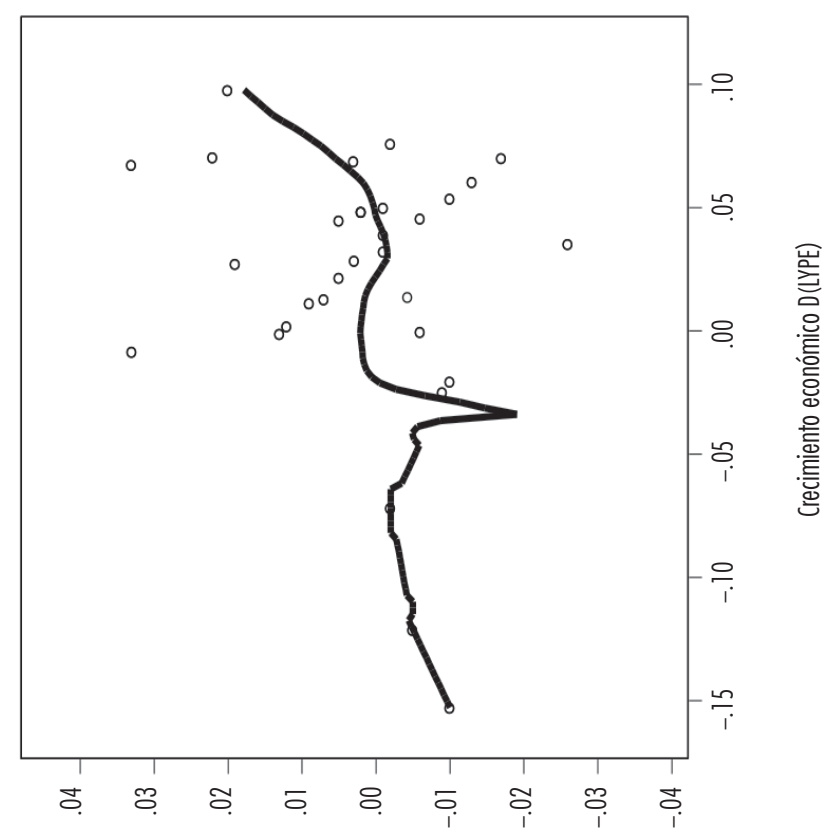

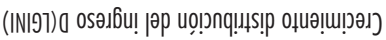

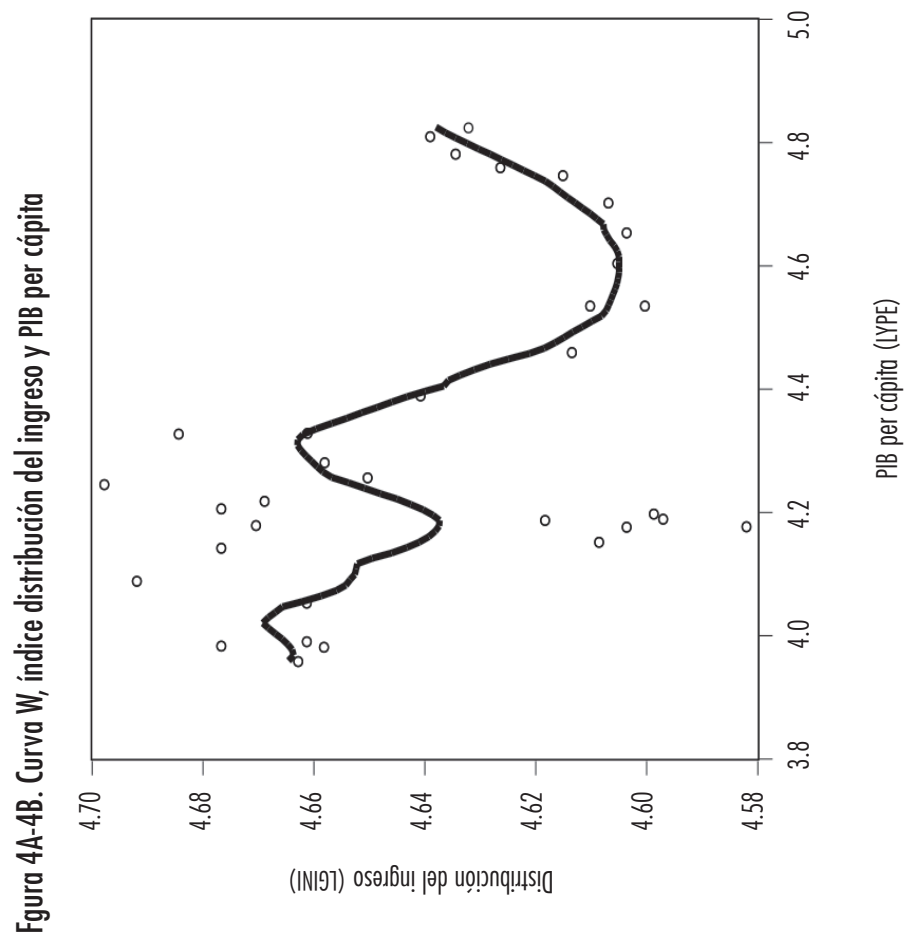

흥
응
흥
흥
흥
0.0
$\overline{0}$
호 


\section{METOdología}

\section{Data}

Se utilizó la tasa de crecimiento del PBI per cápita como crecimiento económico, cuya fuente es el Banco Central de Reservas del Perú (BCRP) y el Banco Mundial (BM). El coeficiente Gini es una variable operacional de la DIP elaborado por el Instituto Nacional de Estadística e Informática (INEI). Algunos autores demuestran que los datos desestiman la verdadera situación de la desigualdad en la DIP, siendo subvalorada (Alarco et al., 2019; Cruz-Saco et al., 2018; Mendoza et al., 2011). El modelo estimado es una serie de tiempo estimada a partir de Mendoza et al. (2011) y se empalma con Cruz-Saco et al. (2018) у вм (2018).

Los coeficientes de Gini (CG) del INEI subestiman la desigualdad por excluir a los hogares de altos ingresos. La Encuesta Nacional de Hogares (ENAHO) es compartida con el Bм que, al igual que el INEI, utiliza la distribución sin corregir por el residual faltante de la cola alta. Los CG reportados por el Bм también subestiman la desigualdad (Cruz-Saco et al., 2018). Característica que identifica Mendoza et al. (2011) y coincide con el hallazgo encontrado por Alarco et al. (2019), Figueroa y Webb (1975) y Yamada et al. (2016). Mendoza et al. (2011) utiliza la metodología de Figueroa (1993) para determinar la DI.

Cruz-Saco et al. (2018) considera que el ingreso ENAHO representa los primeros nueve deciles de la Di. El residual es el ingreso del decil superior (no representado por ENAHO). De esta forma, se obtienen 12 categorías de ingreso, diez deciles, $1 \%$ superior y $5 \%$ superior neto del $1 \%$ superior. Luego, la estimación de este autor es considerada para determinar la serie de tiempo, ya que toma en cuenta los niveles más altos de los hogares ricos y son los datos más actuales encontrados para el periodo 1997-2015. Los datos de Mendoza et al. (2011) se agregan según las tasas de crecimiento para el periodo 1985-1996. Los dos últimos datos consideran BM para el periodo 2016-2017 (вм, 2018).

\section{Método}

El modelo teórico matemático se basa en variables identificadas en la revisión de la literatura y la evidencia empírica, que analizan los determinantes de la DIP, tomando en consideración las causalidades teóricas. La hipótesis es que el lento crecimiento económico no sostenido, explica la desigual Dip durante el periodo 1985-2017, y que existen otros factores teóricos que determinan 
su comportamiento. Según el análisis, la función teórica de la distribución del ingreso sería:

$$
\begin{gathered}
G I N I_{t}=f\left(Y P E_{t}, Z_{t}\right) \\
(+/-)(-/+)
\end{gathered}
$$

Dónde:

$G I N I_{t}:$ la distribución del ingreso, $Y P E_{t}$ : el nivel de PIB per cápita, y $Z_{t}$ son las variables exógenas y de control: mecanismos de transferencia y políticas tributarias operacionalizado como \% ingresos tributarios del РBI (ING), estructura productiva representada por el sector servicios como porcentaje del PBI (sss), capital humano representado por alumnos en educación secundaria (EDUI) y dotación de RR.NN. representado por rentas por explotación del recurso natural petróleo (RRPP). Además, se considera una variable del Fenómeno enOs (Niño y Niña), operacionalizado por el índice enos Multivariado (MEI) elaborado por la Oficina Nacional de Administración Oceánica y Atmosférica (NOAA, por sus siglas en inglés). Una variable dummy (DUMI) del cambio de estrategia de crecimiento y desarrollo económico a partir de 1991.

A partir de la función teórica (1), se especifica un modelo econométrico considerando las variables determinantes de la variable teórica DIP con su variable operacional Gini. Posteriormente, se consideró un modelo para probar HUK. El modelo econométrico se presenta en la ecuación lineal (2), siguiente:

$$
\begin{aligned}
\ln (G I N I)= & \beta_{0}+\beta_{1} \ln (Y P E)+\beta_{2} \ln (I N G)+\beta_{3} \ln (R R P P) \\
& +\beta_{4} \ln (S S S)+\beta_{5} \ln (E D U 1)+\varepsilon_{t}
\end{aligned}
$$

Para la estimación econométrica de los parámetros del modelo econométrico dado en la ecuación lineal (2), se utilizó el modelo ARDL, que son modelos lineales que analizan series de tiempo, donde las variables dependientes e independientes están relacionadas contemporáneamente y a través de valores rezagados, según Narayan (2004), Pesaran y Shin (1997) y Pesaran et al. (2001). El modelo general ARDL $\left(p, q_{1}, q_{2}, q_{3} \ldots q_{k}\right)$, está dado por: $y_{t}$ que es una variable endógena, $\mathrm{y}_{1}, \mathrm{x}_{2}, \mathrm{x}_{3} \ldots \mathrm{x}_{\mathrm{k}}$, son variables exógenas, expresadas en la ecuación (3). 


$$
y_{t}=\alpha_{0}+a_{1} t+\sum_{i=1}^{p} \psi_{i} y_{t-i}+\sum_{j=1}^{k} \sum_{l j=0}^{q j} \beta_{j, l_{j}} x_{j, t-l j}+\varepsilon_{t}
$$

Dónde: $\varepsilon_{t}$ son las innovaciones, $\alpha_{0}$ es un término constante, $\alpha_{1}$ es el coeficiente asociado con una tendencia lineal, $\psi_{i}$ es el coeficiente asociado a rezagos de $y_{t}, y \beta_{j}$, $l j$ son los coeficientes asociados a rezagos de $k$ regresores $x_{j, t}$ para $j=1,2,3, \ldots, k$.

El punto de partida para las aplicaciones ARDL es la estimación de la dinámica intertemporal. Los modelos ARDL se estiman por MCO con la variable endógena en función de las variables exógenas y rezagos. La combinación óptima se establece entonces como la que minimiza, según el criterio de Schwarz (sc).

El análisis de corto plazo encuentra una relación de cointegración en el largo plazo entre variables exógenas y variable endógena. Se prueba si existe una relación de largo plazo entre las variables, a través de la prueba de límites sobre la importancia de los parámetros en la relación de cointegración del modelo de corrección de errores (CE). Se utiliza una prueba $F$ con la hipótesis nula que las variables no están cointegradas.

Se computa el estadístico de prueba y compara con dos valores críticos asintóticos correspondientes a casos donde las variables son I(0) o I(1). Cuando el estadístico de prueba está por encima del valor crítico superior, rechaza la hipótesis nula $\left(\mathrm{H}_{0}\right)$ y concluye que la cointegración es posible y se puede estimar un modelo de CE.

Para Narayan (2004), las estimaciones del método ARDL son insesgadas y eficientes dado que se puede aplicar a estudios que tienen una muestra pequeña, como el presente; estima los componentes de largo y corto plazo del modelo simultáneamente, eliminando los problemas asociados con las variables omitidas y la autocorrelación; y distingue entre variables endógena y exógenas.

\section{RESULTADOS}

Los resultados sugieren que las variables en primeras diferencias son estacionarias y se pueden aplicar modelos de estimación ARDL. En la tabla 2 se observa el análisis de cointegración de los modelos con la prueba de límites. Se muestra la existencia de una relación de cointegración a largo plazo para los tres modelos. 
Luis Varona-Castillo y Jorge Ricardo Gonzales-Castillo

Tabla 2. Cointegración con la prueba de límites, periodo 1985-2017

\begin{tabular}{|c|c|c|c|c|c|}
\hline \multirow[t]{2}{*}{ Modelo } & \multirow{2}{*}{$\begin{array}{l}\text { Variable } \\
\text { dependiente }\end{array}$} & \multicolumn{2}{|c|}{ Prueba de límites } & \multirow{2}{*}{$\frac{\text { Cointegración }}{C P / L P}$} & \multirow[t]{2}{*}{ Procedimiento } \\
\hline & & F-Estadístico & t-Estadístico & & \\
\hline I & LGINI & $7.19>4.53^{\star \star \star}$ & $-3.91>-3.43^{\text {*** }}$ & $\mathrm{Si}$ & Estimación CEM \\
\hline$\|$ & LGINI & $6.38>4.27^{\star \star \star \star}$ & $-4.01>-3.43^{\star \star \star}$ & $\mathrm{Si}$ & Estimación CEM \\
\hline III & LGINI & $6.18>4.10^{\star \star \star}$ & $-4.37>-3.43^{\star \star \star}$ & $\mathrm{Si}$ & Estimación CEM \\
\hline
\end{tabular}

Notas: ${ }^{\star} ;{ }^{\star \star} ;{ }^{\star \star \star}$ indican significancia estadística al 10\%; 5\% y 1\%. El modelo I utiliza la ecuación (2); el modelo II la ecuación cuadrática, HUK (3) y el modelo III la ecuación cúbica, HCW (4).

Fuente: elaboración propia.

En la tabla 3 se presentan los resultados de la estimación del modelo ARDL a partir de la ecuación (2). En el modelo I existe evidencia de una relación causal directa del PBI per cápita a DIP, siendo significativo estadísticamente al $1 \%$. En el modelo II se contrasta HUK, cumpliendo con los signos esperados y estadísticamente significativos. Sin embargo, al estimar el modelo III se obtiene un caso especial de la curva en forma de W. No se cumple con HuK, lo que permite inferir que la curva W ( $\mathrm{HCW}$ ) refleja a la economía peruana con un lento y no sostenido crecimiento económico, con bajos niveles de capital humano y débil institucionalidad que genera la desigual Dip en forma cíclica.

En el modelo III también se presentan los impactos de una causalidad negativa y estadísticamente significativa del capital humano (educación) y DIP, permitiendo inferir que tienden a mejorar la desigual DIP. Mientras que la dotación de RR.NN., a través de las rentas y el cambio de estructura productiva por la mayor participación de servicios en el PBI, tiende a empeorar la DIP.

Identificada la cointegración se estimó el modelo CEM, cuyos resultados se presentan en tabla 4. Se observa evidencia empírica robusta que apoya la hipótesis de una relación positiva y estadísticamente significativa, lo que implica que, a mayor crecimiento económico, se tiende a una mayor desigualdad de DIP (modelos I y III).

De acuerdo con la evidencia empírica obtenida y criterio de Schwarz, se descartan los modelos I, II y IV, V, quedando el modelo III y el modelo VI, que muestran robusta relación en niveles y tasas de crecimiento entre niveles del PBI per cápita y DIP, comportándose en niveles como si tuviera una curva en forma de W. El modelo elegido supera las pruebas de normalidad, multicolinealidad, significancia de la correlación serial y la prueba de homocedasticidad al 5\% de significancia estadística. 
Tabla 3. Estimación modelo ARDL, periodo 1985-2017

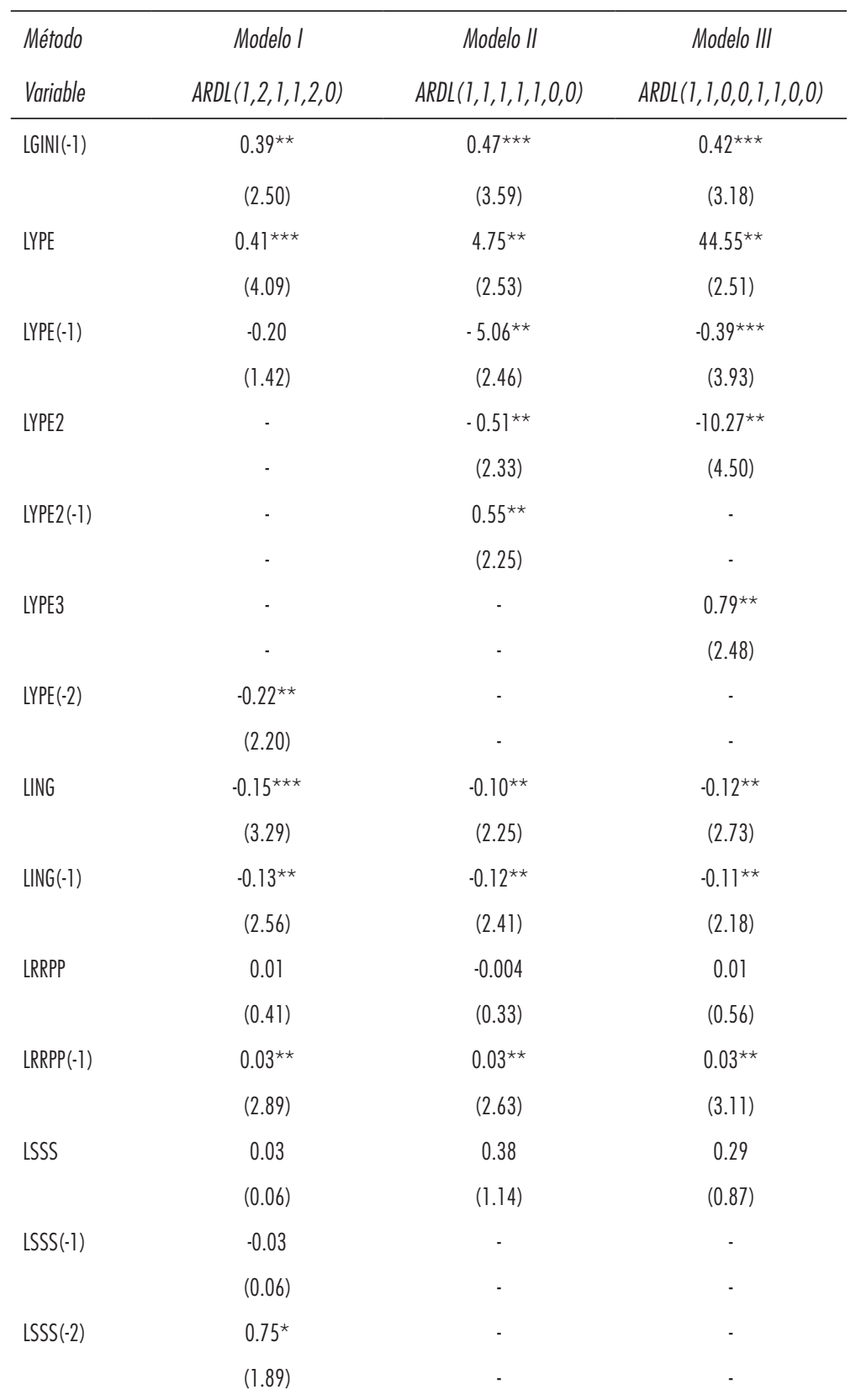

Continúa 
Luis Varona-Castillo y Jorge Ricardo Gonzales-Castillo

Tabla 3. Estimación modelo ARDL, periodo 1985-2017 (continuación)

\begin{tabular}{|c|c|c|c|}
\hline Método & Modelo I & Modelo II & Modelo III \\
\hline Variable & $\operatorname{ARDL}(1,2,1,1,2,0)$ & $\operatorname{ARDL}(1,1,1,1,1,0,0)$ & $\operatorname{ARDL}(1,1,0,0,1,1,0,0)$ \\
\hline \multirow[t]{3}{*}{ LEDUI } & $-0.16^{\star}$ & $-0.19 * \star$ & $-0.16^{\star \star}$ \\
\hline & (2.05) & (2.52) & (2.14) \\
\hline & {$[0.06]$} & {$[0.02]$} & {$[0.05]$} \\
\hline \multirow[t]{2}{*}{ C } & -1.14 & 3.03 & $-61.47^{\star \star}$ \\
\hline & {$[0.46]$} & [0.34] & [2.47] \\
\hline \multirow[t]{2}{*}{ LMEI } & -0.001 & -0.01 & -0.001 \\
\hline & {$[0.93]$} & {$[0.35]$} & {$[0.94]$} \\
\hline \multirow[t]{2}{*}{ DUMI } & 0.03 & 0.01 & 0.03 \\
\hline & {$[0.18]$} & {$[0.65]$} & {$[0.13]$} \\
\hline Klein & $0.89>0.67$ & $0.87>0.67$ & $0.88>0.67$ \\
\hline \multirow[t]{2}{*}{ Jarque-Bera } & $1.56^{\star \star \star}$ & $2.21^{\star \star \star}$ & $0.50^{\star \star \star}$ \\
\hline & {$[0.46]$} & {$[0.33]$} & {$[0.78]$} \\
\hline \multirow[t]{2}{*}{ LM test (l rez) } & $4.36^{\star \star}$ & $4.23^{\star \star}$ & $4.36^{\star \star}$ \\
\hline & {$[0.054]$} & {$[0.055]$} & {$[0.052]$} \\
\hline \multirow[t]{2}{*}{ ARCH test } & 1.64 & 0.09 & 2.28 \\
\hline & {$[0.21]$} & {$[0.76]$} & {$[0.14]$} \\
\hline \multirow[t]{2}{*}{ Ramsey test } & 0.44 & 0.79 & 0.02 \\
\hline & {$[0.52]$} & [0.39] & {$[0.90]$} \\
\hline$R^{2}$ & 0.89 & 0.87 & 0.88 \\
\hline $\mathrm{F}$ & $9.71^{\star \star \star}$ & $9.47^{\star \star \star}$ & $9.98^{\star \star \star}$ \\
\hline Schwarz C. & -4.63 & -4.61 & -4.66 \\
\hline Sample range & 33 & 33 & 33 \\
\hline
\end{tabular}

Notas: un rezago. ( ), estadístico t. [], probabilidad. * ${ }^{\star \star *},{ }^{* * \star}$ indican significancia estadística al 10\%,5\% y $1 \%$. Las pruebas de contraste muestran que los modelos cumplen con la ausencia de un alto grado de multicolinealidad ( $R=0.89 ; 0.87 ; 0.88>0.67$ ), la normalidad ( $p=0.46 ; 0.33 ; 0.78>0.05)$, ausencia de correlación serial $(\mathrm{p}=0.054 ; 0.055 ; 0.052>0.05)$ y heterocedasticidad $(\mathrm{p}=0.21 ; 0.76 ; 0.14>0.05)$. Los modelos están bien especificados al $5 \%$ de significancia (Modelo I, estadístico RESET 0.44; valor $F(1,15)$ y p-valor 0.52 . Modelo II, estadístico RESET 0.79, valor F $(1,17)$ y p-valor 0.38 . Modelo III, estadístico RESET 0.016 ; valor $F(1,17)$ y p-valor 0.90$)$.

Fuente: elaboración propia. 
Tabla 4. Estimación modelo EC (modelo IV, Vy VI), periodo 1985-2017

\begin{tabular}{|c|c|c|c|}
\hline Variable & $\begin{array}{c}\text { Modelo IV } \\
C E(1,2,1,1,2,0)\end{array}$ & $\begin{array}{c}\text { Modelo V } \\
C E(1,1,1,1,1,0,0)\end{array}$ & $\begin{array}{c}\text { Modelo VI } \\
C E(1,1,0,0,1,1,0,0)\end{array}$ \\
\hline \multirow[t]{2}{*}{ dlype } & $0.41^{\star \star \star}$ & $4.75^{\star \star \star}$ & $44.94^{\star \star \star}$ \\
\hline & $(5.70)$ & $(4.15)$ & $(8.32)$ \\
\hline \multirow[t]{2}{*}{ dlype(-1) } & $0.21^{\star \star}$ & - & - \\
\hline & (2.86) & - & - \\
\hline \multirow[t]{2}{*}{ d(lype2) } & - & $-0.51^{\star \star \star}$ & - \\
\hline & - & (3.85) & - \\
\hline \multirow[t]{2}{*}{ dling } & $-0.15^{\star \star \star}$ & $-0.10^{\star \star \star}$ & $-0.11^{\star \star \star}$ \\
\hline & $(5.05)$ & $(3.46)$ & (4.18) \\
\hline \multirow[t]{2}{*}{ dlrrpp } & 0.01 & -0.004 & 0.01 \\
\hline & $(0.65)$ & $(0.52)$ & $(0.98)$ \\
\hline \multirow[t]{2}{*}{ dlsss } & 0.02 & - & - \\
\hline & $(0.09)$ & - & - \\
\hline \multirow[t]{2}{*}{ dlsss $(-1)$} & $-0.74^{\star \star \star}$ & - & - \\
\hline & $(2.94)$ & - & - \\
\hline \multirow[t]{2}{*}{ c } & $1.14^{\star \star \star}$ & $3.03^{\star \star \star}$ & $-61.47^{\star \star \star}$ \\
\hline & $(7.63)$ & $(7.84)$ & (8.28) \\
\hline \multirow[t]{2}{*}{ LMEI } & -0.001 & -0.01 & -0.001 \\
\hline & $(0.15)$ & (1.49) & $(0.12)$ \\
\hline \multirow[t]{2}{*}{ DUM1 } & $0.02^{\star \star}$ & -0.01 & $0.03^{\star \star \star}$ \\
\hline & (2.39) & $(1.09)$ & (3.27) \\
\hline \multirow[t]{2}{*}{$\mathrm{ECM}(-1)$} & $-0.60^{\star \star \star}$ & $-0.52^{\star \star \star}$ & $-0.57^{\star \star \star}$ \\
\hline & (7.52) & (7.72) & (8.28) \\
\hline$R^{2}$ & 0.80 & 0.77 & 0.78 \\
\hline Schwarz & -5.18 & -5.26 & -5.41 \\
\hline $\mathrm{F}$ & $8.54^{\star \star \star}$ & $12.08^{\star \star \star}$ & $15.56^{\star \star \star}$ \\
\hline
\end{tabular}

Fuente: elaboración propia. 
También se observa en la tabla 4, que en todos los modelos la variable del coeficiente de error, ECM (-1) cumple con el signo negativo y son estadísticamente significativos al $1 \%$. Ello implica que las variables del modelo cointegran hasta llegar al equilibrio de largo plazo y en el corto plazo se van corrigiendo a una velocidad de ajuste entre 52 y $60 \%$ anual.

Asimismo, en todos los modelos la tasa de crecimiento en los ingresos tributarios presenta una relación causal negativa tendiendo a mejorar la desigual DIP y es estadísticamente significativa al $1 \%$ (véase tabla 4). Un crecimiento de la participación del sector servicios en el PBI tiene causalidad negativa y permite inferir que también tiende a mejorar DIP, siendo estadísticamente significativo con un rezago. La dotación de RR.NN., operacionalizado por las rentas del petróleo, presenta una relación causal positiva y estadísticamente no significativa, tendiendo a aumentar la desigual DIP, resultado que es coherente con la maldición de los RR.NN., cuya abundancia de RR.NN. tiende a reducir el crecimiento económico. El fenómeno ENOs, que destruye capital físico y humano, no presenta la causalidad esperada y es estadísticamente no significativo. El cambio de estrategia de desarrollo presenta una causalidad negativa, lo que implica que el cambio de una EDISI, donde prevalece el Estado a una EDNL donde prevalece el mercado, ha tendido a empeorar la DIP y es estadísticamente significativa.

\section{DISCUSIÓN}

Se encontró evidencia empírica que apoya la hipótesis que el nivel del PBI per cápita y el crecimiento económico ralentizado y no sostenido de la economía peruana en el periodo 1985-2017, ha empeorado y no ha revertido sostenidamente la desigualdad de DIP al encontrarse una relación de causalidad en forma de W y no una U-invertida de Kuznets.

En la tabla 5 se presentan los resultados de otros trabajos para comparar los coeficientes del ingreso per cápita y educación. El modelo II con coeficientes de 4.75 y -0.51 con los signos esperados y estadísticamente significativos al $5 \%$, coherentes y cercanos a los resultados de Zanzzi et al. (2018), que estima coeficientes tanto para el РвI per cápita como su cuadrado obteniendo 3.08 y -0.17 , que confirma una Hux para Chile. Cheng y Wu (2016) obtienen resultados más altos a los que se encontraron 34.11 y -4.74 , y también confirma una Huk para China.

Nwosa (2019) estima un modelo ARDL con un modelo lineal y el coeficiente del PBI per cápita es 1.15 estadísticamente significativo al 10\% para Nigeria, en comparación al modelo básico estimado que logra 0.41 al 1\% de significancia. 
Tabla 5. Comparación de resultados con evidencia empírica, modelos ARDL

\begin{tabular}{|c|c|c|c|c|c|}
\hline Autor & País & Método & $P I B p c$ & $(P I B p c)^{2}$ & EDU1 \\
\hline \multirow[t]{2}{*}{ Cheng y Wu (2016) } & China & $\begin{array}{l}\operatorname{ARDL}(3,1,0) \\
1981-2011\end{array}$ & $\begin{array}{l}\beta_{1}=25.82^{\star \star \star} \\
p=[0.00]\end{array}$ & $\begin{array}{l}\beta_{2}=-4.01^{\star \star \star} \\
p=[0.00]\end{array}$ & $\begin{array}{l}\beta^{h_{4}}=0.61^{\star * *} \\
p=[0.00]\end{array}$ \\
\hline & & $\begin{array}{l}\operatorname{ARDL}(2,2,0) \\
1981-2011\end{array}$ & $\begin{array}{l}\beta_{1}=34.11^{*} \\
p=(0.06)\end{array}$ & $\begin{array}{l}\beta_{2}=-4.74^{\star} \\
p=(0.063)\end{array}$ & $\begin{array}{l}\beta s_{4}=1.16 \\
p=(0.24)\end{array}$ \\
\hline Zanzzi et al. (2018) & Chile & $\begin{array}{l}\text { ARDL-MCE }(1,0,0,0,0) \\
1984-2013\end{array}$ & $\begin{array}{l}\beta_{1}=3.08^{\star \star \star} \\
\text { ts }=(5.45)\end{array}$ & $\begin{array}{l}\beta_{2}=-0.17^{\star \star \star} \\
\text { ts }=(5.45)\end{array}$ & $\begin{array}{l}\beta h_{4}=0.01 \\
t s=(0.38)\end{array}$ \\
\hline Nwosa (2019) & Nigeria & $\begin{array}{l}\text { ARDL-MCE }(1,1,1,1,1,1) \\
1981-2017\end{array}$ & $\begin{array}{l}\beta_{1}=1.55^{\star} \\
\text { ts }=(2.25) \\
p=[0.05]\end{array}$ & - & - \\
\hline
\end{tabular}

Notas: ${ }^{a}$ utiliza un indicador de desigualdad Theil, con variable proxi de actividad económica: urbanización; $h$, indica educación superior; s, educación secundaria.

Fuente: elaboración propia.

Los hechos estilizados y los modelos ARDL estimados, sugieren una relación de cointegración de largo plazo entre el nivel del PBI per cápita y DIP que lejos de ser una HUK sería HCW, que cae hasta el 2008 y continúa con un nuevo ciclo de crecimiento de DIP a partir de 2010. La relación entre PBI per cápita y la DIP en forma de W presenta coeficientes $\beta_{1}=44.5, \beta_{2}=-10.2$ y $\beta_{3}=0.78$, respectivamente, y son estadísticamente significativos al 5\% (modelo III).

Los resultados muestran que, para Perú, el capital humano (EDUI) tiende a mejorar la DIP, mostrando que la educación o inversión en capital humano es un mecanismo de transmisión de beneficios no sólo para el nivel de ingresos y crecimiento económico, sino también para mejorar la desigual distribución en la DIP, logrando un desarrollo humano, al tender a ser igualadora de ingresos per cápita y de oportunidades. Se obtuvieron coeficientes de -0.16, -0.19 y -0.16 para los tres modelos, respectivamente, y estos resultados coinciden con los de Keller (2012) en Brasil. De acuerdo con Cheng y Wu (2016), el coeficiente de educación secundaria es de 1.16, estadísticamente no significativo, opuesto a lo que se ha encontrado en las estimaciones. Para Zanzzi et al. (2018) encuentran un coeficiente bajo y no significativo (0.01); juzgan que el capital humano no se ha convertido en medio igualador de la DI en Chile.

Se recomienda una agresiva política de inversión en capital humano, privilegiando la calidad en Educación, Investigación, Desarrollo tecnológico, innovación y emprendimiento $(E+I+D+i+e)$. Inversión en parques tecnológicos, 
viveros empresariales e incubadoras de empresas biotecnológicas aplicada a la abundante biodiversidad nacional y promover las innovaciones tecnológicas con sus emprendimientos como la base de un verdadero crecimiento económico endógeno sostenible y con equidad hacia la mejora en la desigual DIP e igualando oportunidades para el logro del desarrollo humano.

Las inversiones en capital humano, vía políticas públicas en salud, reduce enfermedades como son el dengue, la malaria, la anemia y enfermedades virales como el Covid-19 que afectan el capital humano y social (GonzalesCastillo et al., 2020; Varona y Gonzales-Castillo, 2021), impactando positivamente sobre el nivel de ingresos, el crecimiento económico, y tienden a mejorar la desigual DIP. Políticas y reformas dirigidas hacia una economía del conocimiento basada en la producción, distribución, uso del conocimiento e información, configuración territorial y cluster para actuar como vehículo para el crecimiento económico y desarrollo humano (Tello, 2010).

La expansión de ingresos tributarios tiene un impacto negativo y significativo al $1 \%$ reduciendo la desigual DIP. El sistema tributario peruano se caracteriza por ser insuficiente, incompleto, regresivo, inequitativo y no asegura que aporten los que más ingresos y riquezas tengan. Además, las cargas fiscales están distorsionadas o exoneradas a favor de sectores que disfrutan de un trato privilegiado vía estrategias mercantilistas y rentistas de grupos de poder y no se grava impuestos directos sobre ingresos y riqueza. Se recomienda profundizar la Reforma Tributaria con políticas que incrementen la base impositiva vía reducción o eliminación de elusión, evasión o exoneración tributaria y gravar con impuestos al patrimonio generando mayores ingresos para el financiamiento que priorice la inversión en capital humano en salud, educación y seguridad.

El sector servicios tiene un impacto reducidor de la desigual DIP. La estructura productiva se caracteriza por un sector servicios que representa cerca del $60 \%$ del PBI y considera gran parte del $70 \%$ de la mano de obra informal en el país, con bajos niveles de productividad e ingresos. La tendencia en AL y el mundo es a crecer basado en servicios, con elevada productividad e ingresos, ya que representan los dos tercios del valor agregado total mundial (Aboal $e t$ al., 2015). Se recomienda a nivel del Estado promover, capacitar y modernizar dicho sector servicios para aumentar su productividad, dejando de ser un estrato dual y de baja productividad e ingresos (Figueroa, 1993 y 2003), de subempleo e informalidad (Tello, 2011). Asimismo, implementar políticas para diversificar la economía con el desarrollo del turismo, la biodiversidad natural, cultural, gastronómica y de servicios relacionados a las TIC y telecomunicaciones y generar un decrecimiento del tamaño del sector informal. 
Perú posee un gran potencial en RR.NN., generando rentas, canon, regalías mineras y petroleras (CRM). Históricamente dicha riqueza no ha revertido sostenidamente la desigual Dip. Gonzales (2009) demuestra la hipótesis de la maldición por la abundancia de RR.NN. en Perú que se explica por el lento y no sostenido crecimiento económico generado por bajos niveles de capital humano e institucionalidad débil y corrupción. Asimismo, CRM son un mecanismo directo para redistribuir ingresos y descentralizar inversiones en servicios públicos (salud y educación), a través del Gobierno Central (40\%), Regional (25\%) y Local (35\%). Por ende, revertir el impacto directo de CRM sobre la DIP permite recomendar que los contratos con empresas deban considerar el subsidio de las externalidades positivas y la internalización de los daños y las externalidades negativas, así como contribuir a fiscalizar que CRM pueda sustituir capital natural por capital humano fundamentalmente, que busque la rentabilidad socioeconómica y ambiental e invertir en actividades de $E+I+D+i+e$, generadoras de renta futura y sustentabilidad.

Se recomienda promover una nueva institucionalidad que implique que el Gobierno Central, Regional y Local corrijan las "Fallas de Estado", realizando las funciones que le compete por Ley. A nivel de mercado, corregir las "Fallas del Mercado" que implica que los entes reguladores sean fiscalizados y cumplan sus funciones, relacionadas a la promoción de igualdad de oportunidades en la que promueva la competencia empresarial. A la sociedad civil le compete fortalecer la institucionalidad y el capital social en el país.

Las políticas económicas y reformas estructurales por el cambio de estrategia de crecimiento y desarrollo, donde prevalecía el Estado, a una economía de mercado, confirman que tiende a no ser sostenido el crecimiento económico ni la mejora en la Dip. Implica también que el cambio de estrategia de crecimiento y desarrollo ayuda a revertir la tendencia del PBI per cápita y la senda del crecimiento económico a partir de 1992; pero genera un crecimiento económico ralentizado y no sostenido, siendo exógeno al obedecer a la fluctuante y no sostenida demanda internacional de RR.NN. con escaso valor agregado $y$, por ende, la reversión en la desigual DIP tampoco ha sido sostenida.

La desigual DIP, oscila alrededor de etapas ralentizadas de crecimiento económico que depende de los ciclos de los precios internacionales, shocks de oferta, desastres naturales y políticas macroeconómicas, sin el ánimo de lograr en el largo plazo un país con un crecimiento económico sostenible con equidad (Korzeniewicz y Moran, 2005). El proceso no ha operado de forma uniforme y desde la Colonia hasta la actualidad se dan ciclos con periodos de avance intenso seguidos de retrocesos. Ciclos de convergencia y divergencia que son provocados por las relaciones que mantienen distintas regiones y Perú 
con la economía mundial (Seminario y Zegarra, 2016). En la línea de Papanek y Kyn (1986), no es posible encontrar argumentos a favor de HUK, sino que la relación entre el crecimiento económico es dinámica con el crecimiento de la DIP y para economías pequeñas y dependientes como la peruana oscilaría en patrones afectados por los mercados internacionales de materias primas y RR.NN.

Siguiendo a Romer (1990), quien sostiene que la política económica debe priorizar la inversión en capital humano para la innovación. Aplicar capital humano, conocimiento e innovación a los abundantes recursos naturales para dejar de ser una economía primaria-exportadora sujeta al vaivén de los mercados internacionales; coincidiendo con Tello (2011), el crecimiento económico peruano se basa en la explotación y exportación de RR.NN., con capital humano de baja calificación, de bajos niveles de productividad e ingresos y que se caracteriza por no producir el suficiente "efecto goteo", y como consecuencia, la dinámica del crecimiento económico y desarrollo a tasas menores al $2 \%$ promedio anual, que no reduce sostenidamente la desigual DIP. Se recomienda no cambiar el modelo económico, sino todo lo contrario, equilibrar el mercado, el Estado y la sociedad civil, aprovechando la gran dotación de capital natural, sobre todo la biodiversidad, y con inversiones en capital humano en educación que lleve a expandir el mercado interno, con base en servicios y la diversificación productiva con un crecimiento económico sostenible a tasas mayores al 5\% anual que tiendan a mejorar la desigual DIP como un objetivo de desarrollo sostenible.

\section{CONCLUSIONES}

Se encuentra evidencia empírica que apoya la hipótesis que el nivel del PBI per cápita y el crecimiento económico ralentizado y no sostenido de la economía peruana en el periodo 1985-2017 ha empeorado y no ha revertido sostenidamente la desigualdad en DIP reflejada en una relación de causalidad en forma de W, no cumpliéndose Huk y que refleja una economía dual con diferencias de productividades e ingresos con bajos niveles de capital humano y debilidad institucionalidad que genera o mantiene la cíclica y desigual DIP.

Existen otros determinantes que explican de manera causal inversa el crecimiento de la DIP, tendiendo a mejorarla, como son el crecimiento de los ingresos tributarios, la participación del sector servicios en el PBI de altas productividades, la inversión en capital humano, educación, innovación y tecnología; entre otras variables que explica una causalidad directa están la dotación 
de RR.NN., el fenómeno ENos y el cambio de estrategia de crecimiento y desarrollo (EDISI a EDNL).

Es necesario el equilibrio entre mercado, Estado y sociedad civil, con políticas que corrijan efectivamente las fallas de Estado, del mercado y participación de la sociedad civil en el fortalecimiento del capital social e institucional. Son propuestas derivadas de los hallazgos encontrados que van en la dirección de obtener un crecimiento económico sostenido y endógeno que tienda a revertir la desigual Dip y el logro de un desarrollo humano inclusivo y sostenible.

\section{BIBLIOGRAFÍA}

Aboal, D., Arias-Ortiz, E., Crespi, G., Garda, P., Rasteletti, A., Rubalcaba, L., Vairo, M. y Vargas, F. (2015). La innovación y la nueva economía de servicios en América Latina y el Caribe: retos e implicaciones de política. C. y R. Aboal (ed.).

Ahluwalia, M. S. (1976). Income distribution and development: Some stylized facts. The American Economic Review, 66(2). http://www.jstor.org/stable/1817209.

Alarco, G., Castillo, C. y Leyva, F. (2019). Desigualdad factorial, personal y de la riqueza en Perú, 1950-2016. Problemas del Desarrollo. Revista Latinoamericana de Economía, 50(197). http://dx.doi.org/10.22201/ iiec.20078951e.2019.197.67473

Alvaredo, F., Atkinson, A. B., Piketty, T. y Saez, E. (2013). The top 1 percent in international and historical perspective. Journal of Economic Perspectives, 27(3). https://doi.org/10.1257/jep.27.3.3

Banco Mundial (вм) (2018). Índice Gini-Perú. https://datos.bancomundial. org/indicador/SI.POV.GINI?locations=PE

Bourguignon, F. y Morrisson, C. (1990). Income distribution, development and foreign trade: A cross-sectional analysis. European Economic Review, 34(6). https://doi.org/10.1016/0014-2921(90)90071-6

Contreras, D. y Ffrench-Davis, R. (2012). Policy regimes, inequality, poverty and growth: The Chilean experience, 1973-2010. (2012/04). WIDER Working Paper.

Cruz-Saco, M. A., Seminario, B. y Campos, C. (2018). Desigualdad (Re) considerada Perú 1997-2015. Journal of Economics Finance International Business, 2(1). http://dx.doi.org/10.20511/jefib.2018.v2n1.219 
Cheng, W. y Wu, Y. (2016). Understanding the kuznets process-an empirical investigation of income inequality in China: 1978-2011. Social Indicators Research, 134(2). https://doi.org/10.1007/s11205-016-1435-x

Chong, A. y Gradstein, M. (2017). Political and economic inequities and the shaping of institutions and redistribution. Southern Economic Journal, 83(4). https://doi.org/10.1002/soej.12206

De Gregorio, J. y Lee, J. W. (2002). Education and income inequality: New evidence from cross-country data. Review of Income and Wealth, 48(3). https://doi.org/10.1111/1475-4991.00060

Deininger, K. y Squire, L. (1998). New ways of looking at old issues: inequality and growth. Journal of Development Economics, 57(2). https://doi. org/10.1016/S0304-3878(98)00099-6

Figueroa, A. (1973). El impacto de las reformas actuales sobre la distribución de ingresos en el Perú. Apuntes: Revista de Ciencias Sociales, (1). https:// dialnet.unirioja.es/servlet/articulo?codigo $=4998733$

(1993). Crisis distributiva en el Perú. PUCP.

(2003). La sociedad sigma: una teoría del desarrollo económico. PUCP.

(2010). ¿Mejora la distribución del ingreso con la educación? El

caso del Perú. Revista CEPAL, 102. https://repositorio.cepal.org/handle/11362/11422

Figueroa, A. y Webb, R. (1975). Distribución del ingreso en el Perú (1a. ed.). Instituto de Estudios Peruanos.

Foellmi, R. y Zweimüller, J. (2017). Is inequality harmful for innovation and growth? Price versus market size effects. Journal of Evolutionary Economics, 27(2). https://doi.org/10.1007/s00191-016-0451-y

Forbes, K. (2000). A reassessment of the relationship between inequality and growth. American Economic Review, 90(4). https://doi.org/10.1257/ aer.90.4.869

Gonzales-Castillo, J. R. (2009). Recursos naturales y crecimiento económico en la economía peruana [Tesis Doctoral, Universidad Nacional Autónoma de México (UNAM)]. (Reg. 516).

Gonzales-Castillo, J. R., Varona-Castillo, L., Dominguez-Morante, M. G. y Ocaña-Gutierrez, V. R. (2020). Pandemia de la Covid-19 y las Políticas de Salud Pública en el Perú: marzo-mayo 2020. Revista de Salud Pública, 22(2). https://doi.org/10.15446/rsap.v22n2.87373

Guiga, H. y Rejeb, J. B. (2012). Poverty, growth and inequality in developing countries. International Journal of Economics Financial Issues, 2(4). http:// www.econjournals.com 
Heshmati, A. (2004). Growth, inequality and poverty relationships. https://ssrn. $\mathrm{com} / \mathrm{abstract}=602466$

Kaldor, N. (1956). Alternative theories of distribution. The Review of Economic Studies, 23(2). https://www.jstor.org/stable/2296292

Kalecki, M. (1954). Theory of economic dynamics (1a. ed.). Routledge Library Editions.

Keller, Y. (2012). Inequality and economic growth in Brazil [Tesis de Licenciatura]. Universidad de Zurich]. http://www.econ.uzh.ch/ipcdp/theses/ BA_YvesKeller.pdf

Knowles, S. (2005). Inequality and economic growth: The empirical relationship reconsidered in the light of comparable data. The Journal of Development Studies, 41(1). https://doi.org/10.1080/0022038042000276590

Korzeniewicz, R. P. y Moran, T. P. (2005). Theorizing the relationship between inequality and economic growth. Theory and Society, 34(3). https:// doi.org/10.1007/s11186-005-4575-6

Kuznets, S. (1955). Economic growth and income inequality. The American Economic Review, 45(1). https://www.jstor.org/stable/1811581

(1963). Quantitative aspects of the economic growth of nations: VIII. Distribution of income by size. Economic development cultural change, 11(2, Part 2). https://doi.org/10.1086/450006

Lewis, W. A. (1954). Economic development with unlimited supplies of labour. Manchester Sch. Econ. Soc. Stud., 22. https://doi.org/10.1111/j. 1467-9957.1954.tb00021.x

Marshall, A. (1890). Principles of Economic (8a. ed.). McMillan.

Marx, K. (1867). El capital (vol. 1). Siglo XXI.

Mendoza, W., Leyva, J. y Flor, J. L. (2011). La distribución del ingreso en el Perú: 1980-2010. Revista CIS, 9(15). www.untechoparachile.cl/cis

Narayan, P. (2004). Reformulating critical values for the bounds F-statistics approach to cointegration: An application to the tourism demand model for Fiji. https://core.ac.uk/download/pdf/36964011.pdf

Nwosa, P. I. (2019). Income inequality and economic growth in Nigeria: Implication for economic development. https://www.ceeol.com/search/articledetail?id $=854033$

Panizza, U. (2002). Income inequality and economic growth: Evidence from American data. Journal of Economic Growth, 7(1). https://doi. org/10.1023/A:1013414509803

Papanek, G. F. y Kyn, O. (1986). The effect on income distribution of development, the growth rate and economic strategy. Journal of Development Economics, 23(1). https://doi.org/10.1016/0304-3878(86)90079-9 
Pasinetti, L. L. (1962). Rate of profit and income distribution in relation to the rate of economic growth. The Review of Economic Studies, 29(4). http:// www.jstor.org/stable/2296303

Perera-Tallo, F. (2017). Growing income inequality due to biased technological change. Journal of Macroeconomics, 52. https://doi.org/10.1016/j. jmacro.2017.02.002

Pesaran, M. H. y Shin, Y. (1997). An autoregressive distributed-lag modelling approach to cointegration analysis. Paper presented at the Symposium at the Centennial of Ragnar Frisch, The Norwegian Academy of Science and Letters, Oslo, March 3-5, 1995. England. http://citeseerx.ist.psu.edu/ viewdoc/download?doi=10.1.1.153.3246\&rep=rep1\&type=pdf

Shin, Y. y Smith, R. J. (2001). Bounds testing approaches to the analysis of level relationships. Journal of Applied Econometrics, 16(3). https://doi.org/10.1002/jae.616

Piketty, T. (1995). Social mobility and redistributive politics. The Quarterly Journal of Economics, 110(3). https://doi.org/10.2307/2946692

(2014). El capital en el siglo XXI (2a. ed.). Fondo de Cultura Económica.

(2015). About capital in the Twenty-First Century. American Economic Review, 105(5). https://doi.org/10.1257/aer.p20151060

Piketty, T. y Saez, E. (2013). A theory of optimal inheritance taxation. Econometrica, 81(5). https://doi.org/10.3982/ECTA10712

Ranis, G. (1980). Distribución del ingreso y crecimiento en Colombia. Revista Desarrollo y Sociedad, 3. https://revistas.uniandes.edu.co/doi/ pdf/10.13043/dys.3.3

Ranis, G. y Stewart, F. (2002). Crecimiento económico y desarrollo humano en América Latina. Revista CEPAL, 78. https://repositorio.cepal.org/handle/11362/10848

, Stewart, F. y Ramirez, A. (2000). Economic growth and human development. World Development, 28(2). https://doi.org/10.1016/S0305750X(99)00131-X

Ravallion, M. (2001). Growth, inequality and poverty: Looking beyond averages. World Development, 29(11). https://doi.org/10.1016/S0305750X(01)00072-9

Ricardo, D. (1817). On the principles of political economy and taxation. (3a. ed., vol. 31). John Murray.

Romer, P. M. (1990). Endogenous technological change. Journal of Political Economy, 98(5, Part 2). https://doi.org/10.1086/261725 
Seminario, B. y Zegarra, M. A. (2016). La evolución de la desigualdad regional en el Perú, 1795-2007. Centro de Investigación de la Universidad del Pacífico.

Smith, A. (1776). An inquiry into the nature and causes of the wealth of nations Impreso por W. Strahan y T. Cadell.

Tello, M. (2010). Del desarrollo económico nacional al desarrollo local: aspectos teóricos. Revista CEPAL, 102. https://repositorio.cepal.org/handle/ $11362 / 11416$

(2011). Los efectos goteo (trickle down effects) del crecimiento: un análisis del desempeño económico del sector informal a nivel de regiones en el Perú, 2005-2009. En J. M. Iguíniz y J. León (eds.). Desigualdad Distributiva en el Perú. Capítulos Libros PUCP.

Varona, L. y Gonzales-Castillo, J. R. (2021). Dynamics of the impact of COvID-19 on the economic activity of Peru. PLOS ONE, 16(1). https:// doi.org/10.1371/journal.pone.0244920

Wahiba, N. F. y El Weriemmi, M. (2014). The relationship between economic growth and income inequality. International Journal of Economics Financial Issues, 4(1). http://www.acarindex.com/dosyalar/makale/acarindex-1423903663.pdf

White, H. y Anderson, E. (2001). Growth versus distribution: Does the pattern of growth matter? Development Policy Review, 19(3). https://doi. org/10.1111/1467-7679.00134

Yamada, G., Castro, J. F. y Oviedo, N. A. (2016). Revisitando el coeficiente de Gini en el Perú: el rol de las politicas públicas en la evolución de la desigualdad. Documento de discusión ciup (DD1606). Lima.

Zanzzi, P. F., Fernández, J. y González, V. H. (2018). Relación entre la desigualdad en el ingreso, el crecimiento económico, la educación y la pobreza: una explicación desde Kuznets para Chile. Revista Espacios, 39(44). http://www.revistaespacios.com/a18v39n44/a18v39n44p31.pdfpESA 
